\title{
Is Effective of Capsule Endoscopy Compared to Other Diagnostic Modalities in Patients with Small Bowel Crohn's Disease?
}

\author{
Byung Ik Jang \\ Department of Internal Medicine, Yeungnam University College of Medicine, Daegu, Korea
}

See "Effectiveness of Capsule Endoscopy Compared with Other Diagnostic Modalities in Patients with Small Bowel Crohn's Disease: A Meta-Analysis" by Miyoung Choi, et al. on page 62, Vol. 11. No. 1, 2017

Crohn's disease (CD) is a chronic inflammatory disorder associated with both mucosal and transmural inflammation of the bowel wall, involving mainly the small intestine and the colon, but possibly the entire gastrointestinal tract as well as other organs. ${ }^{1}$ Small bowel involvement occurs in about 70\% of CD patients, with up to $30 \%$ patients presenting with disease limited to the ileum. ${ }^{2,3}$ Currently, there is no gold standard for diagnosing small bowel CD. Evaluation of the small intestine with barium radiography is limited by the relative insensitivity for detection of early inflammatory lesions, and endoscopic evaluation is confined to the most distal and proximal small bowel alone.

There are a growing number of reports comparing capsule endoscopy (CE) with other diagnostic tests for use in patients with small bowel CD. CE has been prospectively evaluated in the diagnosis of nonstricturing small bowel $\mathrm{CD}$, as compared with small bowel follow-through (SBFT), ileocolonoscopy (IC), push enteroscopy (PE), enteroclysis (EC), computed tomography enterography (CTE) and magnetic resonance enterography (MRE) with most results showing a significant improvement in diagnostic yield with $\mathrm{CE} .^{4-9}$

Meta-analysis of CE in CD has been conducted previously. Triester et al. ${ }^{2}$ reported that $\mathrm{CE}$ is superior to barium radiography and IC for nonstricturing small bowel CD; whereas, CE showed no significant difference, as compared to alternate diagnostic modalities in patients initially presenting with suspected CD. Dionisio et al. ${ }^{9}$ reported diagnostic yield in patients with suspected and confirmed small-bowel CD, indicating that CE was superior to SBFT, CTE and IC in the evaluation of suspected CD patients. CE was also a more effective diagnostic tool in established CD patients, as compared with SBFT, CTE, and PE.

In this issue of Gut and Liver, Choi et al. ${ }^{10}$ reported results from meta-analysis with published original articles only. After excluding preclinical studies and articles such as reviews, editorials, letters, abstracts, and comment, 24 articles were finally included in the study. Cases were divided into suspected and confirmed $\mathrm{CD}$. The primary outcome was diagnostic yield and diagnostic accuracy of CE. Patient satisfaction, therapeutic impact on treatment, and additional diagnostic information gain in comparison to other modalities were evaluated as secondary outcomes. Quality Assessment of Diagnostic Accuracy Studies-II tool was used to determine the risk of bias. Applicability of concerns for individual studies was graded as low, high, or unclear. Diagnostic modality of detecting lesions of suspected CD was much higher with CE, as compared to SBFT (CE, 66.0\% vs SBFT, 21.3\%; IYw, 0.44; 95\% confidence interval [CI], 0.29 to 0.59; $\mathrm{I}^{2}$, 30\%) and EC (CE, 75.7\% vs EC, 29.4\%; IYw, 0.50; 95\% CI, 0.21 to $\left.0.79 ; \mathrm{I}^{2}, 52 \%\right)$. CE was a more effective diagnostic modality in patients with established CD, as compared with EC (CE, 68.5\% vs EC, 36.7\%; weighted incremental yield, 0.39; 95\% CI, 0.28 to $\left.0.50 ; \mathrm{I}^{2}, 0 \%\right)$. However, it was unclear whether CE was superior to CTE or MRE as a diagnostic tool. CTE and MRE are relatively advanced imaging modalities especially for extraluminal inflammation; and MRE is particularly effective for detecting transmural inflammation, stenosis, and extraluminal lesions such as abscesses and fistula. If patients with known stenosis or stricture were included, the diagnostic yield of CTE and MRE would have been higher than CE, since these can present in up to $25 \%$ of CD cases. CE showed significantly higher diagnostic yield for terminal ileum lesion, as compared to IC, but not CTE.

Correspondence to: Byung Ik Jang

Department of Internal Medicine, Yeungnam University College of Medicine, 170 Hyeonchung-ro, Nam-gu, Daegu 42415, Korea

Tel: +82-53-620-3830, Fax: +82-53-654-8386, E-mail: jbi@med.yu.ac.kr pISSN 1976-2283 eISSN 2005-1212 https://doi.org/10.5009/gnl16549

@) This is an Open Access article distributed under the terms of the Creative Commons Attribution Non-Commercial License (http://creativecommons.org/licenses/by-nc/4.0) which permits unrestricted non-commercial use, distribution, and reproduction in any medium, provided the original work is properly cited. 
With regards to patient's satisfaction, CE was preferred to other modalities due to absence of discomfort during insertion or difficulty in swallowing.

Limitation of this study included no definite criteria for the diagnosis of CD by CE, and various criteria for suspected CD among the studies. Up to $20 \%$ of asymptomatic control patients had nonspecific small bowel abnormalities, suggesting that many findings on CE might be nonspecific or unrelated to $\mathrm{CD}$.

Although CE showed benefits compared to other modalities, routine use of $\mathrm{CE}$ in $\mathrm{CD}$ should be avoided, because capsule retention is a major concern in CD. CE may be helpful in CD patients without stricture, since in such cases, the physician needs to confirm the presence of active disease. CTE or MRE are the preferred modalities for cases of suspected stricture or stenosis.

Large scaled randomized prospective studies are needed to further establish the role of CE for diagnosis and evaluation of suspected small bowel CD.

\section{CONFLICTS OF INTEREST}

No potential conflict of interest relevant to this article was reported.

\section{REFERENCES}

1. Lashner BA. Clinical features, laboratory findings, and course of Crohn's disease. In: Kirsner JB, ed. Inflammatory bowel disease. 5th ed. Philadelphia: Saunders, 2000:305-314.

2. Triester SL, Leighton JA, Leontiadis GI, et al. A meta-analysis of the yield of capsule endoscopy compared to other diagnostic modalities in patients with non-stricturing small bowel Crohn's disease. Am J Gastroenterol 2006;101:954-964.

3. Lee HA, Suk JY, Choi SY, et al. Characteristics of pediatric inflammatory bowel disease in Korea: comparison with EUROKIDS data. Gut Liver 2015;9:756-760.

4. Cheon JH, Kim WH. Recent advances of endoscopy in inflammatory bowel diseases. Gut Liver 2007;1:118-125.

5. Toth E, Fork FT, Almqvist P, et al. Wireless capsule enteroscopy: a comparison with enterography, push enteroscopy and ileocolonoscopy in the diagnosis of small bowel Crohn's disease. Gastrointest Endosc 2004;59:P173.

6. Chong AK, Taylor A, Miller A, Hennessy 0, Connell W, Desmond P. Capsule endoscopy vs. push enteroscopy and enteroclysis in suspected small-bowel Crohn's disease. Gastrointest Endosc 2005;61:255-261.

7. Voderholzer WA, Beinhoelzl J, Rogalla P, et al. Small bowel involvement in Crohn's disease: a prospective comparison of wireless capsule endoscopy and computed tomography enteroclysis. Gut 2005;54:369-373.

8. Albert JG, Martiny F, Krummenerl A, et al. Diagnosis of small bowel Crohn's disease: a prospective comparison of capsule endoscopy with magnetic resonance imaging and fluoroscopic enteroclysis. Gut 2005;54:1721-1727.

9. Dionisio PM, Gurudu SR, Leighton JA, et al. Capsule endoscopy has a significantly higher diagnostic yield in patients with suspected and established small-bowel Crohn's disease: a metaanalysis. Am J Gastroenterol 2010;105:1240-1248.

10. Choi M, Lim S, Choi MG, Shim KN, Lee SH. Effectiveness of capsule endoscopy compared with other diagnostic modalities in patients with small bowel Crohn's disease: a meta-analysis. Gut Liver 2017;11:62-72. 